\title{
RECOVERY OF A POTENTIAL FROM THE RATIO OF REFLECTION AND TRANSMISSION COEFFICIENTS
}

\author{
Tuncay Aktosun \\ Department of Mathematics and Statistics \\ Mississippi State University \\ Mississippi State, MS 39762, USA \\ Vassilis G. Papanicolaou \\ Department of Mathematics \\ National Technical University of Athens \\ Zografou Campus \\ 157 80, Athens, Greece
}

\begin{abstract}
For the one-dimensional Schrödinger equation, the analysis is provided to recover the potential from the data consisting of the ratio of a reflection coefficient to the transmission coefficient. It is investigated whether such data uniquely constructs a reflection coefficient, the number of bound states, bound-state energies, bound-state norming constants, and a corresponding potential. In all the three cases when there is no knowledge of the support of the potential, the support of the potential is confined to a half line, and the support is confined to a finite interval, various uniqueness and nonuniqueness results are established, the precise criteria are provided for the uniqueness and the nonuniqueness and the degree of nonuniqueness, and the recovery is illustrated with some explicit examples.
\end{abstract}

PACS (2003): 02.30.Zz, 03.65.Nk

Mathematics Subject Classification (2000): 34A55, 34L25, 34L40, 47A40, 81U05

Keywords: Schrödinger equation, Inverse scattering, Recovery of potential

Short title: Potential recovery from ratio of scattering coefficients 


\section{INTRODUCTION}

In this paper we investigate the recovery of the potential of the one-dimensional Schrödinger equation from the data consisting of the ratio of a reflection coefficient to the transmission coefficient. We analyze the cases where the potential has no restriction on its support, half-line support, and compact support. We assume no information about the number of bound states, and in fact we try to recover that number as a part of our inverse problem.

Our work is motivated by the work of Rundell and Sacks [1] where it was shown that a bounded, compactly supported potential with a sufficiently small $L^{2}$-norm is uniquely determined by the corresponding ratio of a reflection coefficient to the transmission coefficient. In our paper we show exactly when such a determination is possible.

Consider the Schrödinger equation

$$
\psi^{\prime \prime}(k, x)+k^{2} \psi(k, x)=V(x) \psi(k, x), \quad x \in \mathbf{R},
$$

where the potential $V$ belongs to the Faddeev class, i.e. it is real valued, measurable, and in $L_{1}^{1}(\mathbf{R})$. Here, $L_{n}^{1}(J)$ denotes the class of measurable functions on an interval $J$ such that $\int_{J} d x\left(1+|x|^{n}\right)|V(x)|$ is finite. The prime is used for the derivative with respect to the spatial coordinate $x$. The scattering solutions to (1.1) behave like $e^{i k x}$ or $e^{-i k x}$ as $x \rightarrow \pm \infty$, and they occur for $k \in \mathbf{R} \backslash\{0\}$. A bound state of (1.1) is a solution that belongs

to $L^{2}(\mathbf{R})$ in the $x$ variable, and it is known [2-7] that the bound states can occur only at certain $k$-values on the positive imaginary axis $\mathbf{I}^{+}$in $\mathbf{C}^{+}$. We use $\mathbf{C}^{+}$for the upper-half complex plane and $\mathbf{I}^{+}:=i(0,+\infty)$; later we will let $\overline{\mathbf{C}^{+}}:=\mathbf{C}^{+} \cup \mathbf{R}$ and $\mathbf{I}^{-}:=i(-\infty, 0)$. We will use $N$ to denote the number of bound states, which is known to be finite when $V$ is in the Faddeev class, and suppose that the bound states occur at $k=i \kappa_{j}$ with the ordering $0<\kappa_{1}<\cdots<\kappa_{N}$.

Among the scattering solutions to (1.1) are $f_{\mathrm{l}}$ and $f_{\mathrm{r}}$, the Jost solutions from the left and right, respectively, satisfying the respective boundary conditions

$$
e^{-i k x} f_{1}(k, x)=1+o(1), \quad e^{-i k x} f_{1}^{\prime}(k, x)=i k+o(1), \quad x \rightarrow+\infty,
$$




$$
e^{i k x} f_{\mathrm{r}}(k, x)=1+o(1), \quad e^{i k x} f_{\mathrm{r}}^{\prime}(k, x)=-i k+o(1), \quad x \rightarrow-\infty
$$

From the spatial asymptotics

$$
\begin{array}{ll}
f_{1}(k, x)=\frac{e^{i k x}}{T(k)}+\frac{L(k) e^{-i k x}}{T(k)}+o(1), & x \rightarrow-\infty \\
f_{\mathrm{r}}(k, x)=\frac{e^{-i k x}}{T(k)}+\frac{R(k) e^{i k x}}{T(k)}+o(1), & x \rightarrow+\infty,
\end{array}
$$

we obtain the transmission coefficient $T$, and the reflection coefficients $L$ and $R$ from the left and right, respectively. It is known [2-7] that

$$
\begin{gathered}
T(-k)=T(k)^{*}, \quad R(-k)=R(k)^{*}, \quad L(-k)=L(k)^{*}, \quad k \in \mathbf{R}, \\
R(k)=-\frac{L(-k) T(k)}{T(-k)}, \quad|T(k)|^{2}+|L(k)|^{2}=1, \quad k \in \mathbf{R},
\end{gathered}
$$

where the asterisk denotes complex conjugation. In general, $R$ and $L$ are defined only for real $k$ values, but $T$ has a meromorphic extension to $\mathbf{C}^{+}$. Each bound state corresponds to a (simple) pole of $T$ in $\mathbf{C}^{+}$and vice versa. Given $|T(k)|$ for $k \in \mathbf{R}$ and the bound-state poles $k=i \kappa_{j}$, one can construct $T$ as $[2-7]$

$$
T(k)=\left(\prod_{j=1}^{N} \frac{k+i \kappa_{j}}{k-i \kappa_{j}}\right) \exp \left(\frac{1}{\pi i} \int_{-\infty}^{\infty} d s \frac{\log |T(s)|}{s-k-i 0^{+}}\right), \quad k \in \overline{\mathbf{C}^{+}}
$$

A potential $V$ in the Faddeev class is said to be generic if $T(0)=0$ and exceptional if $T(0) \neq 0$. Generically we have

$$
\lim _{k \rightarrow 0} \frac{2 i k L(k)}{T(k)}=(-1)^{N}\left|W_{0}\right|+o(1), \quad k \rightarrow 0 \text { in } \mathbf{R}
$$

where the Wronskian $W_{0}:=f_{\mathrm{r}}(0, x) f_{1}^{\prime}(0, x)-f_{\mathrm{r}}^{\prime}(0, x) f_{1}(0, x)$ is a nonzero constant. On the other hand, in the exceptional case we have

$$
\lim _{k \rightarrow 0} \frac{L(k)}{T(k)}=\frac{\gamma_{0}^{2}-1}{2 \gamma_{0}}+o(1), \quad k \rightarrow 0 \text { in } \mathbf{R},
$$

where $\gamma_{0}:=\frac{f_{\mathrm{l}}(0, x)}{f_{\mathrm{r}}(0, x)}=(-1)^{N}\left|\frac{f_{\mathrm{l}}(0, x)}{f_{\mathrm{r}}(0, x)}\right|$ is a nonzero constant. 
A potential in the Faddeev class is uniquely determined from the data $\left\{L,\left\{\kappa_{j}\right\},\left\{c_{\mathrm{r} j}\right\}\right\}$ or $\left\{L,\left\{\kappa_{j}\right\},\left\{\gamma_{j}\right\}\right\}$ by using any one of the available methods [2-7]. Here, $c_{\mathrm{r} j}$, for each $j=1, \ldots, N$, represents the bound-state norming constant and $\gamma_{j}$ the dependency constant associated with $k=i \kappa_{j}$, and they are related to the Jost solutions as

$$
c_{\mathrm{r} j}:=\left[\int_{-\infty}^{\infty} d x f_{\mathrm{r}}\left(i \kappa_{j}, x\right)^{2}\right]^{-1 / 2}, \quad \gamma_{j}:=\frac{f_{1}\left(i \kappa_{j}, x\right)}{f_{\mathrm{r}}\left(i \kappa_{j}, x\right)} .
$$

Given $V$, we can remove [2-7] all the bound states from the scattering coefficient and construct the resulting potential $V^{[0]}$ corresponding to the transmission coefficient $T^{[0]}$ and the left reflection coefficient $L^{[0]}$, where

$$
T(k)=T^{[0]}(k) \prod_{j=1}^{N} \frac{k+i \kappa_{j}}{k-i \kappa_{j}}, \quad L(k)=(-1)^{N} L^{[0]}(k) \prod_{j=1}^{N} \frac{k+i \kappa_{j}}{k-i \kappa_{j}} .
$$

The potential $V^{[0]}$ belongs to the Faddeev class whenever $V$ is in that class.

Without loss of any generality, our main problem can be reduced to the recovery of a potential $V$ in the Faddeev class from the data $\mathcal{D}(k):=L(k) / T(k)$ in the following cases:

(i) $V$ has no restrictions on its support.

(ii) The support of $V$ is confined to a half line.

(iii) The support of $V$ is confined to the finite interval.

Our paper is organized as follows. In Section II we study case (i); we see that our data cannot say anything about the value of $N$ in the exceptional case and hence $N \in\{0,1,2, \ldots\}$, and for each such $N$ we have a $2 N$-parameter family of potentials corresponding to our data. On the other hand, in the generic case from our data we get $N \in\{0,2,4, \ldots\}$ or $N \in\{1,3,5, \ldots\}$, as indicated in (1.6); for each allowed $N$ we again have a $2 N$-parameter family of potentials corresponding to $\mathcal{D}$. Case (ii) is analyzed in Section III, and we show that our data puts a further restriction on the maximum allowable values for $N$ and that $N-1$ cannot exceed the number of zeros of $\mathcal{D}$ on $\mathbf{I}^{+}$. In particular, in the generic case where $\mathcal{D}$ has no zeros on $\mathbf{I}^{+}$and the limit in (1.6) is positive, we conclude that $N=0$ and hence there is a unique potential corresponding to $\mathcal{D}$. We also 
show that our data restricts the (open) intervals in which the $\kappa_{j}$ can occur, depending on the sign of $\mathcal{D}$ on $\mathbf{I}^{+}$. Then, for each allowed $N$ we obtain an $N$-parameter family of potentials supported on the positive half line corresponding to our data. We illustrate the nonuniqueness with some explicit examples. Finally, in Section IV, we analyze case (iii) and show that our data further puts severe restrictions on the locations of the $\kappa_{j}$. In this case we show that for each allowed $N$, there can exists only a finite number of potentials corresponding to the same $\mathcal{D}$. We provide the exact criteria for the uniqueness as well as the nonuniqueness and the degree of nonuniqueness, and we illustrate the theory with some explicit example

\section{RECOVERY WITH NO RESTRICTION ON THE SUPPORT}

We will analyze the construction of $V$ from the data $\mathcal{D}$ by analyzing the construction of $\left\{L,\left\{\kappa_{j}\right\},\left\{c_{\mathrm{r} j}\right\}\right\}$ from $\mathcal{D}$.

Given $\mathcal{D}(k)$ for $k \in \mathbf{R}$, we can construct $T^{[0]}$. This is because, as seen from (1.9) and the second equation in (1.4), we have

$$
\frac{1}{\left|T^{[0]}(k)\right|^{2}}=\frac{1}{|T(k)|^{2}}=1+|\mathcal{D}(k)|^{2}, \quad k \in \mathbf{R},
$$

and hence, (1.5) and (2.1) imply that

$$
T^{[0]}(k)=\exp \left(\frac{1}{2 \pi i} \int_{-\infty}^{\infty} d s \frac{\log \left(1 /\left[1+|\mathcal{D}(s)|^{2}\right]\right)}{s-k-i 0^{+}}\right), \quad k \in \overline{\mathbf{C}^{+}} .
$$

Having found $T^{[0]}$, from (1.9) we get

$$
L^{[0]}(k)=(-1)^{N} \mathcal{D}(k) T^{[0]}(k), \quad L(k)=\mathcal{D}(k) T^{[0]}(k)\left(\prod_{j=1}^{N} \frac{k+i \kappa_{j}}{k-i \kappa_{j}}\right) .
$$

Thus, to construct $L$ from $\mathcal{D}$, we must know both the number of bound states and the bound-state energies. In the generic cases, as (1.6) implies, from $\mathcal{D}$ we are only able to determine whether $N$ is even or odd, but in the exceptional case even this is not possible, as implied by (1.7). For $N=0$, as seen from (2.3), $\mathcal{D}$ uniquely determines $L$ and hence also $V$. 
In summary, given the data $\mathcal{D}$, we get, for each value of $N$, a $2 N$-parameter family of corresponding potentials, where $\left\{\left\{\kappa_{j}\right\},\left\{c_{\mathrm{r} j}\right\}\right\}$ represents the parameter set. If $\mathcal{D}(k)$ is bounded at $k=0$ then $N$ can be any nonnegative integer; if $\mathcal{D}(k)$ is unbounded at $k=0$, then $N$ is a nonnegative integer, which is odd or even depending on the sign of $\lim _{k \rightarrow 0}[2 i k \mathcal{D}(k)]$ as in $(1.6)$.

Example 2.1 Let us demonstrate that we can tell from $\mathcal{D}$ whether $N$ is even or odd in the generic case, but not in the exceptional case. Consider

$$
\mathcal{D}(k)=\frac{\alpha}{\left(k+i c_{+}\right)\left(k+i c_{-}\right)}, \quad c_{ \pm}:=\sqrt{10 \pm \sqrt{\alpha^{2}+36}},
$$

where $\alpha \in[-8,8]$ is a parameter. Note that $\alpha= \pm 8$ corresponds to the generic case and $\alpha \in(-8,8)$ corresponds to the exceptional case. In the generic case from (1.6) we get $2 i k \mathcal{D}(k)=\alpha / \sqrt{5}+O(k)$ as $k \rightarrow 0$, and hence $N$ must be even if $\alpha=8$ and odd if $\alpha=-8$.

On the other hand, in the exceptional case, from (1.7) we get $\mathcal{D}(k)=\alpha / \sqrt{64-\alpha^{2}}+O(k)$ as $k \rightarrow 0$, and $N$ can be any nonnegative integer. In fact, the corresponding scattering coefficients for $\alpha \in[-8,8]$ are given by

$$
L(k)=\frac{(-1)^{N} \alpha}{(k+2 i)(k+4 i)}\left(\prod_{j=1}^{N} \frac{k+i \kappa_{j}}{k-i \kappa_{j}}\right), \quad T(k)=\frac{\left(k+i c_{+}\right)\left(k+i c_{-}\right)}{(k+2 i)(k+4 i)}\left(\prod_{j=1}^{N} \frac{k+i \kappa_{j}}{k-i \kappa_{j}}\right),
$$

where $L(0)=-1$ and $T(0)=0$ are assured in the generic case by the choice $N \in$ $\{0,2,4, \ldots\}$ if $\alpha=8$ and $N \in\{1,3,5, \ldots\}$ if $\alpha=-8$.

\section{RECOVERY WITH SUPPORT ON A HALF LINE}

In this section we analyze the construction of $V$ from $\mathcal{D}$ when we further know that the support of $V$ is confined to a half line. Equivalently, we analyze the construction of $\left\{L,\left\{\kappa_{j}\right\},\left\{c_{\mathrm{r} j}\right\}\right\}$ from our data.

There is no loss of generality in assuming that the support of $V$ is confined to $\mathbf{R}^{+}$. This can be argued as follows. If the support of the potential is known to be confined to the interval $(a,+\infty)$ for some real constant $a$, then the value of $a$ can be extracted [8] from $\mathcal{D}$; the shift $V(x) \mapsto V(x-a)$ results in $L(k) / T(k) \mapsto L(k) e^{2 i k a} / T(k)$ and hence there 
is no loss of generality in assuming that $a \equiv 0$. On the other hand, if the support of the potential is known to be confined to $\mathbf{R}^{-}$, then, because of the first equation in (1.4), our problem can also be formulated as the recovery of $V$ from $R / T$, which is equivalent to the recovery of $V$ from $\mathcal{D}$.

When the support of $V$ is confined to $\mathbf{R}^{+}$, it is already known [9-14] that $L$ uniquely determines $V$. In fact, the meromorphic extension of $L(k)$ from $\mathbf{R}$ to $\mathbf{C}^{+}$uniquely determines $\left\{\left\{\kappa_{j}\right\},\left\{c_{\mathrm{r} j}\right\}\right\}$ as indicated in Theorem 3.3 below. Thus, the number of arbitrary parameters appearing in the reflection coefficient $L$ constructed from our data is the same as the number of parameters appearing in the constructed $V$.

In Section II we have seen that $\mathcal{D}$ in the generic case reveals whether $N$ is even or odd. We will next show that knowledge that the support of $V$ is confined to $\mathbf{R}^{+}$leads to an upper bound on $N$ both in the generic and exceptional cases.

Proposition 3.1 Assume $V^{[0]}$ is a potential in the Faddeev class, has support in $\mathbf{R}^{+}$, and has no bound states. Suppose $V$ is the potential obtained by adding $N$ successive bound states to $V^{[0]}$ at $k=i \kappa_{j}$ with $0<\kappa_{1}<\cdots<\kappa_{N}$, and let $L^{[0]}$ denote the left reflection coefficient for $V^{[0]}$ as in (1.9). If the support of $V$ is confined to $\mathbf{R}^{+}$, then $(-1)^{j} L^{[0]}\left(i \kappa_{j}\right)>0$ for $j=1, \ldots, N$, or equivalently, $(-1)^{N-j} \mathcal{D}\left(i \kappa_{j}\right)>0$.

PROOF: If $V \equiv 0$ for $x<0$, from (1.2) and (1.3) we see that

$$
f_{\mathrm{r}}(k, x)=e^{-i k x}, \quad f_{1}(k, x)=\frac{e^{i k x}}{T(k)}+\frac{L(k) e^{-i k x}}{T(k)}, \quad x \leq 0 .
$$

Hence, using $1 / T\left(i \kappa_{j}\right)=0,(3.1)$, and the second equation in (1.8), we conclude that $\gamma_{j}=(L / T)\left(i \kappa_{j}\right)$. Then, with the help of $(1.9)$, we get $L^{[0]}\left(i \kappa_{j}\right)=(-1)^{N} \gamma_{j} T^{[0]}\left(i \kappa_{j}\right)$. It is already known that $T^{[0]}(k)>0$ on $\mathbf{I}^{+}$and $(-1)^{N-j} \gamma_{j}>0$. Thus, $(-1)^{j} L^{[0]}\left(i \kappa_{j}\right)>0$ for $j=1, \ldots, N$. With the help of $\mathcal{D}(k)=(-1)^{N} L^{[0]}(k) / T^{[0]}(k)$, we equivalently claim that $(-1)^{N-j} \mathcal{D}\left(i \kappa_{j}\right)>0$.

For $k \in \mathbf{I}^{+}$we know that $L^{[0]}(k)$ is real valued and continuous and $1 / T^{[0]}(k)>0$; hence, from Proposition 3.1 we obtain the following result.

Corollary 3.2 Assume $V$ belongs to the Faddeev class, has $N$ bound states, and has support 
in $\mathbf{R}^{+}$. Let $L^{[0]}$ denote the left reflection coefficient for $V^{[0]}$, which is obtained from $V$ by removing all the bound states, as in (1.9). Then, $L^{[0]}$ must have at least $N-1$ zeros on $\mathbf{I}^{+}$, equivalently, $\mathcal{D}$ must have at least $N-1$ zeros on $\mathbf{I}^{+}$.

Let us note that the zeros of $L^{[0]}$ on $\mathbf{I}^{+}$, or equivalently those of $\mathcal{D}$, need not be simple, as indicated in Examples 3.8 and 3.9.

The following theorem gives a characterization of the left reflection coefficient corresponding to a potential in the Faddeev class with support confined to $\mathbf{R}^{+}$. Let

$$
\hat{L}(\alpha):=\frac{1}{2 \pi} \int_{-\infty}^{\infty} d k L(k) e^{i k \alpha}, \quad \hat{R}(\alpha)=:-\frac{1}{2 \pi} \int_{-\infty}^{\infty} d k \frac{L(-k) T(k)}{T(-k)} e^{i k \alpha} .
$$

Theorem 3.3 The left reflection coefficient $L$ corresponds to a unique potential $V$ in the Faddeev class with support in $\mathbf{R}^{+}$and with $N$ bound states at $k=i \kappa_{j}(j=1, \ldots, N)$ if and only if the following conditions hold:

(i) $L$ is continuous on $\mathbf{R}$, and $L(-k)=L(k)^{*}$ for $k \in \mathbf{R}$.

(ii) $|L(k)| \leq 1-C k^{2} /\left(1+k^{2}\right)$ on $\mathbf{R}$ for some positive constant $C$.

(iii) $L(0) \in[-1,1)$.

(iv) L has a meromorphic extension to $\mathbf{C}^{+}$with $N$ simple poles occurring at $k=i \kappa_{j}$ and residues $\operatorname{Res}\left[L\left(i \kappa_{j}\right)\right]=i c_{\mathrm{r} j}^{2}$ for some positive constants $c_{\mathrm{r} j}$. Of course, if $N=0$ then the extension of $L$ to $\mathbf{C}^{+}$is analytic there.

(v) $L(k)=o(1 / k)$ as $k \rightarrow \infty$ in $\overline{\mathbf{C}^{+}}$.

(vi) The function $k / T(k)$, where $T(k)$ is given in (1.5) with $|T(s)|=\sqrt{1-|L(s)|^{2}}$, is continuous in $\overline{\mathbf{C}^{+}}$.

(vii) The functions $\hat{L}$ and $\hat{R}$ defined in (3.2) are absolutely continuous, $\hat{L}^{\prime} \in L_{1}^{1}(-\infty, 0)$, and $\hat{R}^{\prime} \in L_{1}^{1}(a,+\infty)$ for any $a<0$.

PROOF: The proof is obtained by modifying the characterization conditions on the scattering data $[5,7,15]$ corresponding to a potential in the Faddeev class in order to take into account the vanishing property of the potential on $\mathbf{R}^{-}$. It is known [9-14] that (iv) is equivalent to vanishing of $V$ on $\mathbf{R}^{-}$. The slight modification in (vii) is also related to the 
vanishing of the potential on $\mathbf{R}^{-}$.

In the following we illustrate the recovery of $V$, or equivalently of $L$, by presenting some explicit examples.

Example 3.4 Let our data for a potential with support in $\mathbf{R}^{+}$be given by

$$
\mathcal{D}(k)=\frac{-\sqrt{3}(k-i)(k-3 i)}{(k+i)^{2}(k+\sqrt{5} i)(k+3 i)} .
$$

Notice that $\mathcal{D}(k)$ is bounded at $k=0$ and hence this corresponds to an exceptional case. Proceeding as in (2.2), or equivalently by solving the Riemann-Hilbert problem [cf. (2.1)]

$$
\frac{1}{T^{[0]}(-k)}=\left(1+|\mathcal{D}(k)|^{2}\right) T^{[0]}(k), \quad k \in \mathbf{R},
$$

we obtain

$$
T^{[0]}(k)=\frac{(k+i)(k+\sqrt{5} i)}{(k+\sqrt{2} i)(k+2 i)}
$$

From (2.3) we get

$$
L^{[0]}(k)=(-1)^{N} A(k), \quad A(k):=\frac{-\sqrt{3}(k-i)(k-3 i)}{(k+i)(k+\sqrt{2} i)(k+2 i)(k+3 i)} .
$$

Notice that $\mathcal{D}$ has two zeros on $\mathbf{I}^{+}$. Hence, the number of bound states of $V$ cannot exceed 3. Since this is the exceptional case, $N$ is allowed to be any of $0,1,2$, and 3 . Recalling the fact that $L$ uniquely determines $V$ because of the support property of $V$, with the help of the sign restriction indicated in Proposition 3.1, or equivalently, with the help of Theorem 3.3(iv), we obtain all the following possibilities for $L$ and also for $V$.

(a) For $N=0$, we have $L(k)=A(k)$, and the potential $V$ is uniquely determined.

(b) For $N=1$, we have $L(k)=A(k) \frac{k+i \kappa_{1}}{k-i \kappa_{1}}$, where $\kappa_{1} \in(0,1) \cup(3,+\infty)$ is the only arbitrary parameter in $V$.

(c) For $N=2$, we have $L(k)=A(k) \prod_{j=1}^{2} \frac{k+i \kappa_{j}}{k-i \kappa_{j}}$, where $\kappa_{1} \in(1,3)$ and $\kappa_{2} \in(3,+\infty)$ are the only two arbitrary parameters in $V$.

(d) For $N=3$, we have $L(k)=A(k) \prod_{j=1}^{3} \frac{k+i \kappa_{j}}{k-i \kappa_{j}}$, where $\kappa_{1} \in(0,1), \kappa_{2} \in(1,3)$, and $\kappa_{2} \in(3,+\infty)$ are the only three arbitrary parameters in $V$. 
Example 3.5 Let $\mathcal{D}(k)=\frac{8}{k(k+i \sqrt{20})}$. This is the generic case because $\mathcal{D}(k)$ is singular at $k=0$. Using $(2.1)$ we get $T^{[0]}(k)=\frac{k(k+i \sqrt{20})}{(k+2 i)(k+4 i)}$. From (1.6) we see that $\lim _{k \rightarrow 0}[2 i k \mathcal{D}(k)]=8 / \sqrt{5}$, which is positive, and hence $N \in\{0,2,4, \ldots\}$. Then, from the first equation in $(2.3)$ we get $L^{[0]}(k)=\frac{8}{(k+2 i)(k+4 i)}$. Since $\mathcal{D}$ has no zeros on $\mathbf{I}^{+}$, Corollary 3.2 implies that the only possibility is $N=0$. Thus, $L(k)=L^{[0]}(k)$, and our data uniquely determines $L$ and $V$.

Example 3.6 Let $\mathcal{D}(k)=\frac{-8}{k(k+i \sqrt{20})}$. As in Example 3.5, this is the generic case and $T^{[0]}(k)=\frac{k(k+i \sqrt{20})}{(k+2 i)(k+4 i)}$. From (1.6) we see that $\lim _{k \rightarrow 0}[2 i k \mathcal{D}(k)]=-8 / \sqrt{5}$, which is negative, and hence $N \in\{1,3,5, \ldots\}$. Then, from the first equation in (2.3) we get $L^{[0]}(k)=\frac{8}{(k+2 i)(k+4 i)}$. Since $\mathcal{D}$ has no zeros on $\mathbf{I}^{+}$, Corollary 3.2 implies that the only possibility is $N=1$. Thus, we get $L(k)=-L^{[0]}(k) \frac{k+i \kappa_{1}}{k-i \kappa_{1}}$, where $\kappa_{1} \in(0,+\infty)$ is an arbitrary parameter. Because the constructed $L$ contains one arbitrary parameter, there exists a one-parameter family of potentials corresponding to our data.

Example 3.7 Let $\mathcal{D}(k)=\frac{-8(k-3 i)(k-4 i)}{k(k+i \sqrt{20})(k+3 i)(k+4 i)}$. As in Example 3.5, this is the generic case and $T^{[0]}(k)=\frac{k(k+i \sqrt{20})}{(k+2 i)(k+4 i)}$. From (1.6) we see that $\lim _{k \rightarrow 0}[2 i k \mathcal{D}(k)]=$ $-8 / \sqrt{5}$, which is negative, and hence $N \in\{1,3,5, \ldots\}$. Then, as in Example 3.5 we get $L^{[0]}(k)=\frac{8(k-3 i)(k-4 i)}{(k+2 i)(k+3 i)(k+4 i)^{2}}$. Since $\mathcal{D}$ has two zeros on $\mathbf{I}^{+}, N$ cannot exceed 3 . Thus, we must have $N=1$ or $N=3$. In conjunction with Proposition 3.1 or Theorem 3.3(iv), for $N=1$ we get the one-parameter family $L(k)=-L^{[0]}(k) \frac{k+i \kappa_{1}}{k-i \kappa_{1}}$ with $\kappa_{1} \in(0,3) \cup$ $(4,+\infty)$; on the other hand, for $N=3$ we get $L(k)=-L^{[0]}(k) \prod_{j=1}^{3} \frac{k+i \kappa_{j}}{k-i \kappa_{j}}$ with $\kappa_{1} \in(0,3)$, $\kappa_{2} \in(3,4)$, and $\kappa_{3} \in(4,+\infty)$. Thus, our data corresponds to a one-parameter family of potentials when $N=1$, and it corresponds to a three-parameter family of potentials when $N=3$.

Example 3.8 Let $\mathcal{D}(k)=\frac{8(k-i)^{2}}{k(k+i \sqrt{20})(k+i)^{2}}$. We see that $\mathcal{D}$ has a single double zero 
on $\mathbf{I}^{+}$. As in Example 3.5 we get

$$
T^{[0]}(k)=\frac{k(k+i \sqrt{20})}{(k+2 i)(k+4 i)}, \quad L^{[0]}(k)=\frac{8(k-i)^{2}}{(k+2 i)(k+4 i)(k+i)^{2}} .
$$

Note that $\lim _{k \rightarrow 0}[2 i k \mathcal{D}(k)]=8 / \sqrt{5}$, which is positive, and hence (1.6) implies that $N \in$ $\{0,2,4, \ldots\}$. On the other hand, Corollary 3.2 implies that $N=0$ or $N=2$; however, a sign analysis of $\mathcal{D}$ on $\mathbf{I}^{+}$indicates that $\mathcal{D}(i \beta)<0$ for $\beta \in(0,1) \cup(1,+\infty)$ and $\mathcal{D}(i \beta)=0$ for $\beta=1$. Thus, $N=2$ is incompatible with Proposition 3.1. Hence, $N=0$ is the only possibility, and $T$ and $L$ are uniquely determined by our data as equal to $T^{[0]}$ and $L^{[0]}$, respectively, given in (3.3). Therefore, there exists a unique potential corresponding to our data.

Example 3.9 Let $\mathcal{D}(k)=\frac{-8(k-i)^{2}}{k(k+i \sqrt{20})(k+i)^{2}}$. The corresponding $T^{[0]}$ and $L^{[0]}$ are the same as in (3.3). From (1.6) we see that $\lim _{k \rightarrow 0}[2 i k \mathcal{D}(k)]=-8 / \sqrt{5}$, which is negative, and hence $N \in\{1,3,5, \ldots\}$, as implied by (1.6). A sign analysis of $\mathcal{D}$ on $\mathbf{I}^{+}$indicates that $\mathcal{D}(i \beta)>0$ for $\beta \in(0,1) \cup(1,+\infty)$ and $\mathcal{D}(i \beta)=0$ for $\beta=1$. Hence, with the help of Corollary 3.2 we conclude that $N=1$ is the only possibility. We thus obtain

$$
T(k)=\frac{k(k+i \sqrt{20})\left(k+i \kappa_{1}\right)}{(k+2 i)(k+4 i)\left(k-i \kappa_{1}\right)}, \quad L(k)=\frac{-8(k-i)^{2}\left(k+i \kappa_{1}\right)}{(k+2 i)(k+4 i)(k+i)^{2}\left(k-i \kappa_{1}\right)},
$$

with $\kappa_{1} \in(0,1) \cup(1,+\infty)$. Therefore, our data corresponds to a one-parameter family of potentials, where $\kappa_{1}$ acts as the parameter.

\section{RECOVERY WITH COMPACT SUPPORT}

In this section we analyze the recovery of $V$, or equivalently of $L$, from $\mathcal{D}$ when it is further known that the support of $V$ is confined to a finite interval. In constructing $\left\{L,\left\{\kappa_{j}\right\},\left\{c_{\mathrm{r} j}\right\}\right\}$ from $\mathcal{D}$, all the results obtained in Sections II and III are certainly valid in this section as well. We have the following:

(i) In the generic case, we are able to tell via (1.6) whether the nonnegative integer $N$ representing the number of bound states of $V$ is even or odd.

(ii) Using (2.2) and the second equation in (2.3), we are able to construct $T^{[0]}$ and determine $L$ except perhaps for the values of $\kappa_{1}, \ldots, \kappa_{N}$. 
(iii) Let us use $Z$ to denote the number of zeros of $\mathcal{D}$ on $\mathbf{I}^{+}$. From Corollary 3.2 we conclude that $N \leq Z+1$. Moreover, Proposition 3.1 imposes a further restriction on $N$ depending on the sign of $\mathcal{D}$ on $\mathbf{I}^{+}$.

(iv) The quantity $T^{[0]}$, which is uniquely determined by $\mathcal{D}$, has a meromorphic extension to the entire complex plane due to the fact that the support of $V$ is confined to a finite interval. We will show that the set $\left\{-i \kappa_{j}\right\}$ has to be a subset of the set of zeros of $1 / T^{[0]}$ on $\mathbf{I}^{-}$.

The following result is already known [2], and hence its proof is omitted. By writing the first equation in (1.9) as $\frac{k}{T(k)} \prod_{j=1}^{N} \frac{1}{k-i \kappa_{j}}=\frac{k}{T^{[0]}(k)} \prod_{j=1}^{N} \frac{1}{k+i \kappa_{j}}$, which is valid on the entire complex plane, the reader can compare the zeros of $1 / T^{[0]}(k)$ and of $1 / T(k)$ on the imaginary axis and verify the result stated in (iv) above as well as those in following proposition.

Proposition 4.1 Assume $V^{[0]}$ is real-valued, is integrable, has support confined to a finite interval, and has no bound states. Suppose $V$ is the potential obtained by adding $N$ successive bound states to $V^{[0]}$ at $k=i \kappa_{j}$ with $0<\kappa_{1}<\cdots<\kappa_{N}$, and let $T^{[0]}$ and $T$ denote the transmission coefficients for $V^{[0]}$ and $V$, respectively. If the support of $V$ is confined to a finite interval, then $k / T^{[0]}(k)$ and $k / T(k)$ are both entire, $1 / T^{[0]}$ has a simple zero at $k=-i \kappa_{j}$ for $j=1, \ldots, N$, and any other zero of $1 / T^{[0]}$ on $\mathbf{I}^{-}$must also be a zero $1 / T$ with the same multiplicity.

In the first example below, we show that not every zero of $1 / T^{[0]}$ on $\mathbf{I}^{-}$necessarily corresponds to a bound state of $V$. In the second example we illustrate the recovery of $L$ and $V$ from our data.

Example 4.2 Consider the square-well potential supported on the interval [0,1] with depth equal to $c \alpha^{2}$ for some $c, \alpha>0$. The corresponding transmission coefficient satisfies $\frac{1}{T(k)}=e^{i k}\left[\cos \gamma+\frac{k^{2}+\gamma^{2}}{2 i k \gamma} \sin \gamma\right]$ with $\gamma:=\sqrt{k^{2}+c \alpha^{2}}$. It can be easily checked that $1 / T(-i \alpha)=0$ if we choose $\alpha=\ln 8=2.0794 \overline{4}$ and $c=8 / 9$, where the overline on a digit indicates a roundoff. With these values, $V$ has exactly one bound state occurring at $k=i \kappa$ 
with $\kappa=1.3078 \overline{2}$. We have $1 / T(i \alpha) \neq 0$ and $1 / T(i \kappa)=1 / T^{[0]}(-i \kappa)=0$. In other words, $k=i \alpha$ does not correspond to a bound state of $V$ even though $1 / T^{[0]}(-i \alpha)=0$.

Example 4.3 Let $\mathcal{D}(k)=\frac{-\epsilon e^{i k} \sin \sqrt{k^{2}+\epsilon}}{2 i k \sqrt{k^{2}+\epsilon}}$, where $\epsilon$ is a nonnegative parameter. In fact, one corresponding potential is the square well of depth $\epsilon$ supported on the interval $[0,1]$. For each value of $\epsilon$, let us obtain all the potentials corresponding to $\mathcal{D}(k)$ with support confined to a finite interval. We have $\lim _{k \rightarrow 0}[2 i k \mathcal{D}(k)]=-\sqrt{\epsilon} \sin \sqrt{\epsilon}$, and hence the exceptional case occurs when $\sqrt{\epsilon}=p \pi$ for $p=0,1, \ldots$ and the generic case occurs when $\sqrt{\epsilon} \neq p \pi$. In the generic case we see that the sign of $\lim _{k \rightarrow 0}[2 i k \mathcal{D}(k)]$ is that of $(-1)^{p+1}$ when $p \pi<\sqrt{\epsilon}<(p+1) \pi$, and hence we can tell from $\epsilon$ whether $p$ is even or odd. The sign analysis of $\mathcal{D}$ on $\mathbf{I}^{+}$shows that $Z$ mentioned in (iii) in the beginning of this section is equal to $\lfloor\sqrt{\epsilon} / \pi\rfloor$, i.e. the greatest integer less than or equal to $\sqrt{\epsilon} / \pi$; in other words, $\mathcal{D}$ has $Z$ zeros on $\mathbf{I}^{+}$occurring at $k=i z_{j}$ with $z_{j}=\sqrt{\epsilon-(j-1)^{2} \pi^{2}}$ for $j=1, \ldots, Z$. In this particular example, $\mathcal{D}$ happens to have $Z$ zeros on $\mathbf{I}^{-}$as well occurring at $k=-i z_{j}$ symmetrically located with respect to the origin. With the help of (2.1) and (2.2) we obtain

$$
\frac{1}{T^{[0]}(k)}=e^{i k}\left[\cos \sqrt{k^{2}+\epsilon}+\frac{2 k^{2}+\epsilon}{2 i k \sqrt{k^{2}+\epsilon}} \sin \sqrt{k^{2}+\epsilon}\right] \prod_{j=1}^{Z+1} \frac{k+i \beta_{j}}{k-i \beta_{j}},
$$

where the $\left\{\beta_{j}\right\}$ is the ordered set with $0<\beta_{1}<\cdots<\beta_{Z+1}$ consisting of those positive $\beta$ values satisfying $\tan \sqrt{\epsilon-\beta^{2}}=\frac{2 \beta \sqrt{\epsilon-\beta^{2}}}{\epsilon-2 \beta^{2}}$. According to (iii) we must have $N \leq Z+1$. Using all these constraints, we can determine all the possibilities for $N$, the corresponding bound states, reflection coefficient $L$, and potential $V$. For example, we have the following:

(a) When $\epsilon=5$, the above analysis shows that $Z=0$ and thus $N \leq 1$, we are in the generic case and $N$ must be odd, the quantity $1 / T^{[0]}$ given in (4.1) has one simple zero on $\mathbf{I}^{-}$at $k=-i \beta_{1}$, where $\beta_{1}=1.585 \overline{7}$. Thus, we must have $N=1$ with the bound state occurring at $k=i \beta_{1}$, and

$$
L(k)=\mathcal{D}(k) T^{[0]}(k) \frac{k+i \beta_{1}}{k-i \beta_{1}}=\frac{-\epsilon \tan \sqrt{k^{2}+\epsilon}}{2 i k \sqrt{k^{2}+\epsilon}+\left(2 k^{2}+\epsilon\right) \tan \sqrt{k^{2}+\epsilon}} .
$$

(b) When $\epsilon=10$, we find that $Z=1$ with $z_{1}=0.36110 \overline{2}$, and thus $N \leq 2$, we are in the generic case and $N$ must be even, the quantity $1 / T^{[0]}$ given in (4.1) has two simple 
zeros on $\mathbf{I}^{-}$at $k=-i \beta_{j}$, where $\beta_{1}=0.32442 \overline{2}$ and $\beta_{2}=2.5475 \overline{9}$. Thus, we have either of the two cases where $N=0$ or $N=2$. For $N=0$, we get $L(k)=\mathcal{D}(k) T^{[0]}(k)$. On the other hand, for $N=2$ we get $L(k)=\mathcal{D}(k) T^{[0]}(k) \frac{\left(k+i \beta_{1}\right)\left(k+i \beta_{2}\right)}{\left(k-i \beta_{1}\right)\left(k-i \beta_{2}\right)}$.

(c) When $\epsilon=50$, we find that we are in the generic case, $N$ must be odd, $Z=2$ with $z_{1}=3.243 \overline{7}$ and $z_{2}=6.3348 \overline{6}$, and thus $N \leq 3$; moreover, the quantity $1 / T^{[0]}$ given in (4.1) has four simple zeros on $\mathbf{I}^{-}$at $k=-i \beta_{j}$, where $\beta_{1}=1.871 \overline{5}, \beta_{2}=5.1983 \overline{9}$, $\beta_{3}=5.4264 \overline{9}$, and $\beta_{4}=6.637 \overline{6}$. Thus, we have either of the two cases where $N=1$ or $N=3$. For $N=1$ there is double nonuniqueness with $L(k)=\mathcal{D}(k) T^{[0]}(k) \frac{k+i \beta_{1}}{k-i \beta_{1}}$ or $L(k)=\mathcal{D}(k) T^{[0]}(k) \frac{k+i \beta_{4}}{k-i \beta_{4}}$, which is a consequence of $\mathcal{D}\left(i \beta_{1}\right)>0, \mathcal{D}\left(i \beta_{2}\right)<0$, $\mathcal{D}\left(i \beta_{3}\right)<0$, and $\mathcal{D}\left(i \beta_{4}\right)>0$. For $N=3$ we again have double nonuniqueness with the three bound states occurring at $k=i \kappa_{j}$ with the ordered set $\left\{\kappa_{1}, \kappa_{2}, \kappa_{3}\right\}$ being equal to either $\left\{\beta_{1}, \beta_{2}, \beta_{4}\right\}$ or $\left\{\beta_{1}, \beta_{3}, \beta_{4}\right\}$.

(d) When $\epsilon=100$, we find that we are in the generic case, $N$ must be even, $Z=3$ with $z_{1}=3.3426 \overline{9}, z_{2}=7.778 \overline{7}$, and $z_{3}=9.4937 \overline{9}$, and thus $N \leq 4$; moreover, the quantity $1 / T^{[0]}$ given in (4.1) has six simple zeros on $\mathbf{I}^{-}$at $k=-i \beta_{j}$, where $\beta_{1}=1.9269 \overline{3}$, $\beta_{2}=5.7103 \overline{8}, \beta_{3}=6.4101 \overline{4}, \beta_{4}=8.5460 \overline{7}, \beta_{5}=9.1847 \overline{6}$, and $\beta_{6}=9.6526 \overline{2}$. Thus, we have either of the three cases where $N=0, N=2$, or $N=4$. For $N=0$ our data uniquely determines $L$ and $V$, with $L(k)=\mathcal{D}(k) T^{[0]}(k)$. For $N=2$, with $L(k)=\mathcal{D}(k) T^{[0]}(k) \prod_{j=1}^{2} \frac{k+i \kappa_{j}}{k-i \kappa_{j}}$, we have five-fold nonuniqueness where the two bound states occurring at $k=i \kappa_{j}$ with the ordered set $\left\{\kappa_{1}, \kappa_{2}\right\}$ being equal to either of $\left\{\beta_{1}, \beta_{2}\right\},\left\{\beta_{1}, \beta_{3}\right\},\left\{\beta_{1}, \beta_{6}\right\},\left\{\beta_{4}, \beta_{6}\right\}$, and $\left\{\beta_{5}, \beta_{6}\right\}$. On the other hand, for $N=4$ we have four-fold nonuniqueness where the four bound states occurring at $k=i \kappa_{j}$ with the ordered set $\left\{\kappa_{1}, \kappa_{2}, \kappa_{3}, \kappa_{4}\right\}$ being equal to either of $\left\{\beta_{1}, \beta_{2}, \beta_{4}, \beta_{6}\right\},\left\{\beta_{1}, \beta_{2}, \beta_{5}, \beta_{6}\right\}$, $\left\{\beta_{1}, \beta_{3}, \beta_{4}, \beta_{6}\right\},\left\{\beta_{1}, \beta_{3}, \beta_{5}, \beta_{6}\right\}$.

Acknowledgments. The research leading to this article was supported in part by the National Science Foundation under grant DMS-0204437 and the Department of Energy under grant DE-FG02-01ER45951. 


\section{REFERENCES}

[1] Rundell, W. and Sacks, P., "On the determination of potentials without bound state data," J. Comput. Appl. Math. 55, 325-347 (1994).

[2] Faddeev, L. D., "Properties of the $S$-matrix of the one-dimensional Schrödinger equation," Amer. Math. Soc. Transl. (Ser. 2) 65, 139-166 (1967).

[3] Deift, P. and Trubowitz, E., "Inverse scattering on the line," Comm. Pure Appl. Math. 32, 121-251 (1979).

[4] Newton, R. G., "The Marchenko and Gel'fand-Levitan methods in the inverse scattering problem in one and three dimensions," in Conference on inverse scattering: theory and application, edited by J. B. Bednar, R. Redner, E. Robinson, and A. Weglein (SIAM, Philadelphia, 1983), pp. 1-74.

[5] Marchenko, V. A., Sturm-Liouville operators and applications (Birkhäuser, Basel, 1986).

[6] Chadan, K. and Sabatier, P. C., Inverse problems in quantum scattering theory, 2nd ed. (Springer, New York, 1989).

[7] Aktosun, T. and Klaus, M., "Chapter 2.2.4, Inverse theory: problem on the line," in Scattering, edited by E. R. Pike and P. C. Sabatier (Academic Press, London, 2001), pp. $770-785$.

[8] Portinari, J. C., "An inverse scattering transform for potentials of compact support," J. Math. Phys. 19, 2100-2102 (1978).

[9] Novikova, N. N. and Markushevich, V. M., "Uniqueness of the solution of the onedimensional problem of scattering for potentials located on the positive semiaxis," Comput. Seismology 18, 164-172 (1987).

[10] Aktosun, T., Klaus, M., and van der Mee, C., "On the Riemann-Hilbert problem for the one-dimensional Schrödinger equation," J. Math. Phys. 34, 2651-2690 (1993).

[11] Aktosun, T., "Bound states and inverse scattering for the Schrödinger equation in one 
dimension," J. Math. Phys. 35, 6231-6236 (1994).

[12] Grébert, B. and Weder, R., "Reconstruction of a potential on the line that is a priori known on the half line," SIAM J. Appl. Math. 55, 242-254 (1995).

[13] Aktosun, T., "Inverse Schrödinger scattering on the line with partial knowledge of the potential," SIAM J. Appl. Math. 56, 219-231 (1996).

[14] Gesztesy, F. and Simon, B., "Inverse spectral analysis with partial information on the potential. I. The case of an a.c. component in the spectrum," Helv. Phys. Acta 70, 66-71 (1997).

[15] Melin, A., "Operator methods for inverse scattering on the real line," Comm. Partial Differential Equations 10, 677-766 (1985). 